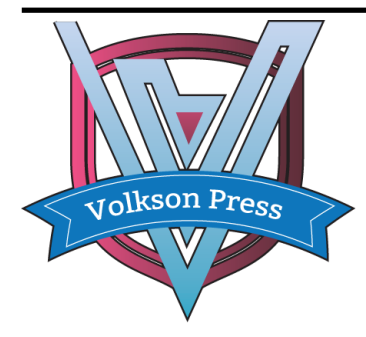

Contents List available at VOLKSON PRESS

\title{
A SIMPLE AND EFFECTIVE ROUTE FOR PREPARATION OF POLYSTYRENE(PS) NANOSPHERE CONTAINING CDSE QUANTUM DOTS BY ELECTROSPINNING
}

\author{
Song Yan*, Wang Fang-Fei \\ Department of Materials Science and Engineering, Jilin institute of Chemical Technology, Chengde Street, Jilin, China. \\ *Corresponding Author Email: songyan199809@163.com
}

This is an open access article distributed under the Creative Commons Attribution License, which permits unrestricted use, distribution, and reproduction in any medium, provided the original work is properly cited

\section{ARTICLE DETAILS}

\section{Article History:}

Received 26 June 2018 Accepted 2 July 2018

Available online 1 August 2018

\section{ABSTRACT}

Fluorescent CdSe quantum dots (QDs) were dispersed into polystyrene (PS) nanospheres by electrospinning a CdSe/PS blent solution. In the process, assembled quantum dots did not take place, quantum dots were uniformly dispersed in the polymer matrix which were either single-colored or double-colored nanospheres. The morphology and distribution of composite nanospheres were characterized by field emission scanning electron microscopy (FESEM) and transmission electron microscopy (TEM).The diameter of the composite nanospheres is reduced from $1150 \mathrm{~nm}$ of a pure PS nanosphere to about $570 \mathrm{~nm}$ with the increased amount of CdSe QDs. The photoluminescence (PL) spectral analysis indicates that the peak position of CdSe QDs composite nanospheres in either solid state or water is identical to that of CdSe/PS blending solution, at about $587 \mathrm{~nm}$ and $611 \mathrm{~nm}$.especially the interference possibility of two peaks in double-colored system is avoided. And the position is still unchangeable when they are left for at least one month, indicating the stable photoluminescent property of the fluorescent composite nanospheres.

\section{KEYWORDS}

Polystyrene (PS), CdSe QDs, fluorescent, composite nanosphere, electrospinning.

\section{INTRODUCTION}

As a new type of wavelength conversion material, semiconductor quantum dots have excellent characteristics such as narrow emission spectrum, high quantum yield, and easy integration with devices. It can adjust their optical and electrical properties by changing the size $[1,2]$. These excellent characteristics have made it possible to have significant potential applications in different fields such as lasers, LEDs, photovoltaic devices, photoelectric sensors, biological detection, and have received widespread attention [3]. QDs can also be linked with biorecognition molecules such as peptides, antibodies, nucleic acids, and be used as fluorescent probes [4-6]. With the rapid development of genomics and proteomics, esecially the publication of finished human genic atlas and produced massively parallel data, there is the need for technologies that can rapidly screen a large number of nucleic acids and proteins [7]. QDs offer several unique advantages during the course, such as size- and composition-tunable emission from visible to infrared wavelengths, large absorption coefficients across a wide spectral range, and very high levels of brightness and photostability [8]. One-dimensional (1D) nanostructures, such as various nanoparticles, nanorods, nanotubes, nanowires, and nanospheres, have received much attention in recent years and have emerged as a powerful class of building blocks to construct nanoscale photonic and electronic devices $[9,10]$. If QDs can be introduced into 1D nanostructures, it will open a new era in the application of nanocomposites to electronic and optoelectronic devices [11-14]. However, embedding QDs in a 1D polymer is not easy, especially embedding QDs in submicrometer- and nanometer-sized fibers with dramatically high aspect ratios. Since QDs tend to aggregate and their properties are ultrasensitive to environmental variations, the most challenging thing is the homogeneous distribution of QDs in polymer nanospheres with preservation of their optical properties.There has been a report about the preparation method of QDsbeads. First of all, polymer beads were obtained mainly through suspension or emulsion polymerization, then the followed operation of mixing and stiring helped to embed QDs in polymer beads [15-17]. However, this method usually results in a waste of QDs. The electrospinning has been considered to be a convenient, effective, and versatile technique for generating materials with diameters ranging from several micrometers to tens of nanometers since its rise in recent decades $[18,19]$.

Recently,inorganic hybrid (organic/inorganic) compounds and some nanomaterials with special structures have also been prepared by electrospinning. As an efficient, economical and reliable technology, electrospinning technology has been widely used in the preparation of various ultra-fine fibers. Due to its prepared fiber diameter can reach between tens of nanometers to several microns [20]. Thus, the luminescent microspheres obtained through electrospinning combining the merits of electrospinning will be of great significance in both its preparation and developing technology $[21,22]$.

In this work, with polystyrene (PS) and CdSe QDs as materials,both singlecolored and double-colored CdSe/PS QDs composite nanospheres were fabricated by electrospinning. The morphology and structure of nanospheres were characterized by field emission scanning electron microscopy (SEM) and Transmission electron microscopy (TEM). Their photoluminescence (PL) propertyies were analysed with the aid of fluorescence spectra.

\section{EXPERIMENTAL}

\subsection{Materials}

Polystyrene (PS, M=8,000,000) was supplied by Xiamen Sanland Chemicals Company (China), CdSe QDs was supplied by America, N, Ndimethylformamide (DMF) and chloroform were purchased from Beijing Chemicals Co.China. All the chemicals were used without further purification. 


\subsubsection{Preparation of CdSe/PS QDS Electrospinning}

Typically, $0.010 \mathrm{~g}$ of PS was dissolved in $1.0 \mathrm{~g}$ of DMF and stirred for about 12 hours to ensure the dissolution of PS, then a controlled amount of CdSe QDs with wavelength of the emission peak at $587 \mathrm{~nm}$ or $611 \mathrm{~nm}$ CdSe QDs was added into the chloroform to prepare solutions with different concentrations of $2 \%, 4 \%, 6 \%$ respectively, the above two solutions were mixed at a weight ratio of 7:3 ultimately forming singlecolored CdSe/PS blending solutions.Furthermore, both $587 \mathrm{~nm}$ and $611 \mathrm{~nm}$ CdSe QDs in a certain radio were added to chloroform first, and then mixed with the PS solution, double-colored CdSe / PS blending solutions were thus obtained finally. We prepared three groups of CdSe / PS blending solutions in ratios of two types of the CdSe QDs 1:1; 1:2; and 2: 1 respectively.

\subsubsection{Preparation of CdSe/PS QDs composite nanospheres}

The above prepared solutions were respectively placed in a $5 \mathrm{ml}$ glass syringe that was connected to the anode that is $15 \mathrm{~cm}$ far away from Al-foil collector as the cathode. The mixed solution was electrospun at $15.0 \mathrm{KV}$ positive voltage and a flow rate of $0.5 \mathrm{ml} / \mathrm{h}$. Thus CdSe/PS QDs composite nanospheres were obtained.

\subsubsection{Characterization}

The morphologies of the CdSe/PS QDs composite nanospheres collected onto the aluminum plate were determined under an FEI XL30 scanning electron microscope (SEM). PL measurements were performed on an RF-5301PC Fluorescence Spectrophotometer. TEM image was taken via a Hitachi H-8100 electron microscope operated at $200 \mathrm{kV}$ with a CCD

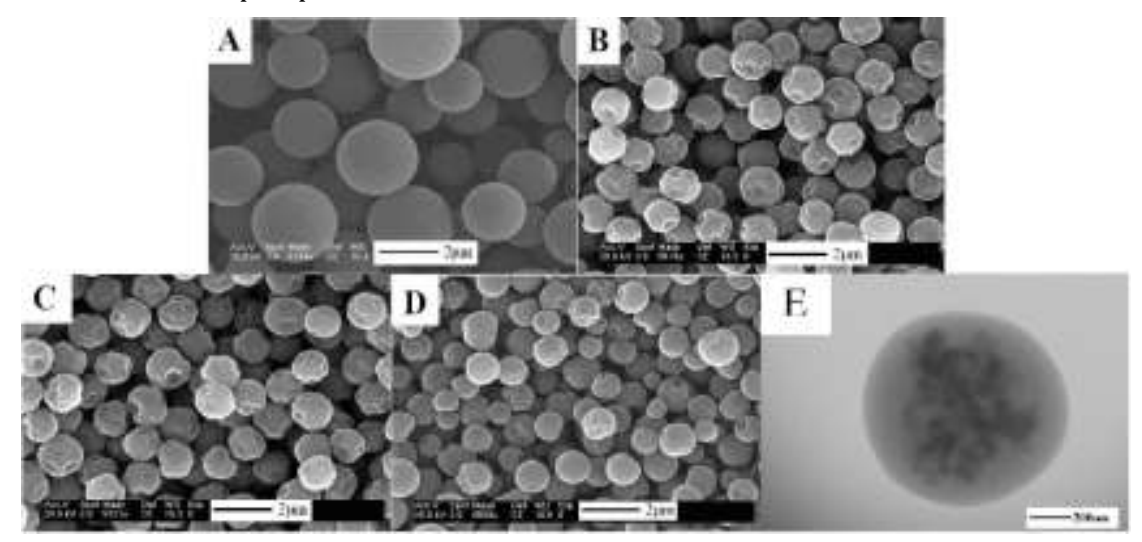

Figure 1: SEM images of pure PS nanospheres(A) and CdSe / PS composite nanospheres with CdSe contents of (wt\%) (B) 2, (C) 4, and (D) 6, TEM images of the CdSe/PS nanoshpheres (E)

Equally, the specific distribution of QDs is revealed by fluorescence spectra too. If the assembled QDs take place, the position of emission peak will be red-shifted. Figure2.(A) and (B) denotes the PL spectra of the different Single-colored CdSe/PS blendinging solutions and composite nanospheres. Compared to that of the CdSe/PS blending solution, the emission peak of the resultant nanospheres hardly changes, located at $587 \mathrm{~nm}$ and $611 \mathrm{~nm}$, indicating a very good separation of the QDs in the nanospheres and no occurrence of energy transfer. This phenomenon is different from that of recent report on a similar system, in which FRET occurred in CdTe-PVA inkjet-printed films. These results clearly reveal that during the electrospinning process, the doped CdSe QDs were

homogeneously and separately dispersed in the PS matrix. Because the camera.

\section{RESULTS AND DISCUSSION}

CdSe/PS composite nanospheres were successfully prepared through electrospinning. In Fig.1.SEM image shows the morphology of PS nanospheres, and the diameters of the nanospheres were generally measured in SEM by comparison with the scale bar. As can be seen from Figure1.(A), the pure PS nanospheres exhibit average diameter of an about $1150 \mathrm{~nm}$. Figure1.(B), (C) and (D) show that the diameters of CdSe/PS nanospheres obviously decreased with increasing concentration of CdSe QDs in the PS solution.When the content of CdSe QDs was $2 \mathrm{wt} \%$, $4 \mathrm{wt} \%$ or $6 \mathrm{wt} \%$ in the solution. The average diameters of the CdSe/PS nanospheres were $730 \mathrm{~nm}, 680 \mathrm{~nm}$ and $570 \mathrm{~nm}$ respectively. In terms of the morphology, the suface of composite nanospheres are not as smooth as that of pure PS nanospheres after adding CdSe QDs to them, concavo-convex surface can be seen from the SEM images. This is because the addition of QD results in the increased conductivity of solutions, the diameter of nanospheres become smaller, the surface charge density of droplets tip is also increased, carried relatively large charge density of a droplet after emitting ultimately increases the droplet instability. In the course of flight, the polymer was stretched by strong power in electric field, resulting in the emergence of a state of non-spherical.

At the same time, in order to verify the dispersion circumstance of CdSe QDs in nanospheres, TEM images of the nanospheres containing CdSe QDs were taken,which show that CdSe QDs appeared as little dark spots inside the nanospheres(Figure1.(E)). These results indicate that CdSe QDs have well-proportioned dispersal in PS nanospheres. process of nanosphere's solidification developed from its initial formation during electrospinning requires a quite short time, there is a possibility that the optical properties of CdSe QDs are well preserved.With the further consideration of the future application of CdSe/PS composite nanospheres in the field of biology, it is therefore necessary to investigate their luminescent property and stability in the humid environment. Accordingly, we dispersed nanospheres in the water, the test result indicates that its peak in the water is still at $587 \mathrm{~nm}$ and $611 \mathrm{~nm}$ as that of them placed in the dry environment. And the optical properties of the samples were measured a month later, it can be seen that only the intensity of the peak is slightly decreased while the peak positions are almost unchangeable, whether the nanospheres are in either solid state or water.
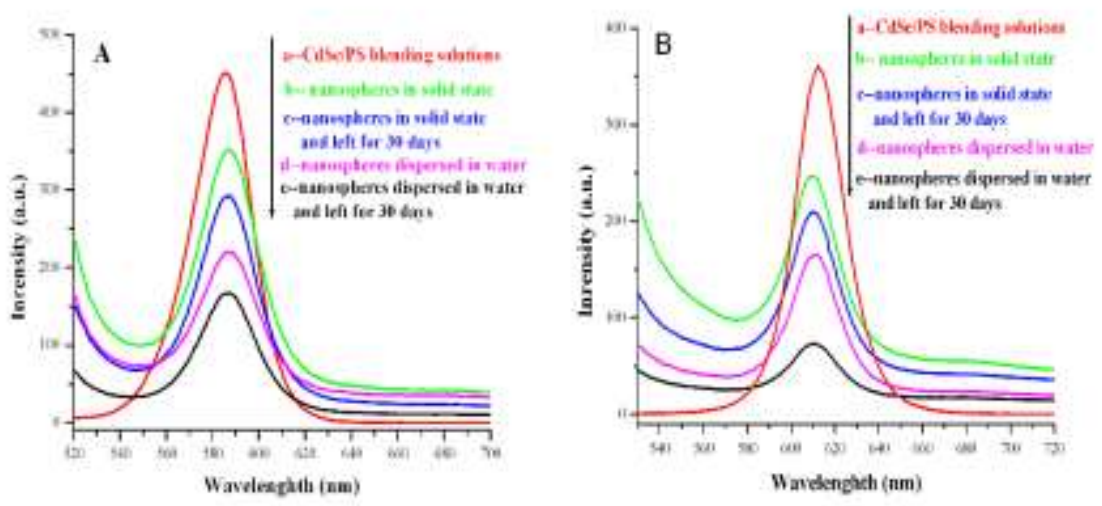

Figure 2: FL spectra of sigle-colored CdSe/PS composite nanospheres emission wavelengths is $587 \mathrm{~nm}(\mathrm{~A}), 611 \mathrm{~nm}(\mathrm{~B})$ 
When CdSe/PS composite nanospheres are practically used, fluorescence composite microspheres of single-component are not enough, because the characterization of a particular signal often needs more participation of the mixed composition of QDs, the occurrence of optical interference between QDs with different emission wavelengths is specially not desired. From this point of view, we have also conducted a further test of doublecolored experiment. Figure.3((A)-(C) is the PL spectra of the CdSe/PS composite nanospheres containing two types of CdSe QDs with different emission wavelengths. There were three groups of solutions in the experiment and each group had a different weight ratio of raw materials which means the luminous intensities of two components in one sample will be different. The results show that as is the case of sigle-colored nanospheres, the positon and appearance of emission peak of doublecolored nanospheres are identical to those of CdSe/PS blending solutions despite of the different weight ratios of raw materials. Optical properties of CdSe QDs are well kept with little change, which demonstrates that QDs were successfully embedded in the PS matrix with well preserved specific nature. All of these advantages discussed above will lay a solid foundation for the application of microspheres in the future.
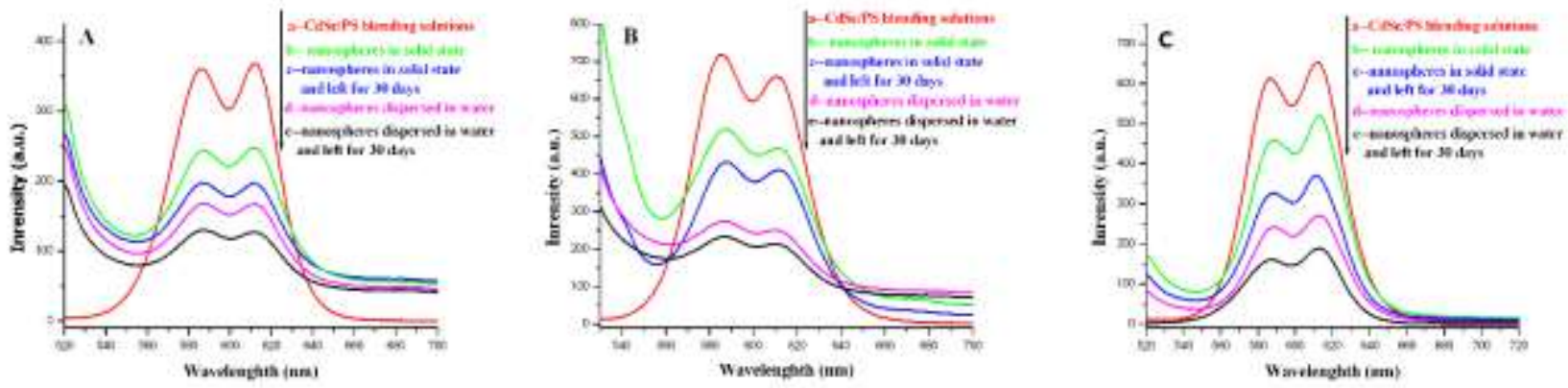

Figure 3: FL spectra of double-colored composite nanospheres with different weight ratios of two types of CdSe QDs 1:1(A), 1:2(B), and 2:1(C)

\section{CONCLUSIONS}

To sum up, CdSe QDs were incorporated in PS nanospheres by electrospinning. In the process, there was no occurrence of QDs assembled, CdSe QDs were dispersed homogeneously in the polymer matrix. We prepared two groups of one-component system and three groups of double-component system of luminescent PS nanospheres respectively. Compared to that of the pure PS nanospheres, the diameter of the CdSe/PS composite nanosphere is significantly reduced from $1150 \mathrm{~nm}$ to $570 \mathrm{~nm}$. However, owing to the blending of CdSe QDs, the surface of the nanospheres is not smooth. The analysis of PL spectra indicates that the peak position of both single-colored and double-colored nano shpheres in either solid state or water is identical to that of CdSe /PS blending solution, at about $586 \mathrm{~nm}$ and $611 \mathrm{~nm}$, a special point worth emphasizediszing is that there is no ocurrence of peak interference in double-colored system. And the peak position is still unchangeable when they are left for at least one month,indicating the stable photoluminescent property of the fluorescent composite nanospheres. All of these will be benificial to the future application of nanospheres in the related research of genomics.

\section{REFERENCES}

[1] Jun, S., Jang, E. 2013. Bright and Stable Alloy Core / Multishell Quantum Dots. Angew. Chem, 125 (2), 707-710.

[2] Ning, P., Zhang, C., Liu, J.G. 2016. Photoluminescence and Thermal Stability of $\mathrm{Mn}^{2+}$-Doped Cdse /Cds /Zns Quantumdots. Proceedings of the 13th China International Forum On Solid State Lighting, 63-65.

[3] Shirasaki, Y., Suprang, J. 2013. Emergence of Colloidal Quantum-Dot Light-Emitting Technologies. Nature, 7, 13-23.

[4] Wang, X., Ding, B. 2011. Precise Structure Control and Applications Of Nanowebs. Nano Today, 6, 510.

[5] Li, Y., Qian, F., Xiang, J., Lieber, C.M. 2006. Nanowire Electronic and Optoelectronic Devices. Materials Today, 9, 18.

[6] Burck, J., Heissler, S., Geckle, U. 2013. Resemblance of Electrospun Collagen Nanofibers to Their Native Structure. Langmuir, 29, 1562.

[7] Gao, X., Cui, Y., Levenson, R.M., Chung, L.W., Nie, S.M. 2004. In Vivo Cancer Targeting and Imaging with Semiconductor Quantum Dots. Nature Biotechnology, 22, 969.

[8] Ganesh, V.A., Nair, A.S. 2012. Superhydrophobic Fluorinated PossPvdf-Hfp Nanocomposite Coating on Glass by Electrospinning. Mater. Chem., 22, 18479.
[9] Freiberg, S., Zhu, X.X. 2004. Polymer Microspheres for Controlled Drug Release. International Journal of Pharmaceutics, 282, 1.

[10] Carlberg, B., Li-Lei, Ye. 2011. Surface-Confined Synthesis Of Silver Nanoparticle Composite Coating On Electrospun Polyimide Nanofibers. Small, 7, 3057.

[11] Sinha, V.R., Bansal, K., Kaushik, R., Trehan, A. 2004. Poly-EpsilonCaprolactone Microspheres and Nanospheres: An Overview. International Journal of Pharmaceutics, 274, 1.

[12] Loewen, P.C., Carpena, X., Rovira, C., Ivancich, A., Pluque, R., Haas, R., Odenbreit, S., Nicholls, P., Fita, I. 2004. Biochemistry, 43, 3089.

[13] Lee, J., Sundar, V.C., Heine, J.R., Bawendi, M.G., Jensen, K.F. 2000. Full Color Emission from Ii-Vi Semiconductor Quantum Dot-Polymer Composites. Adv. Mater, 12, 1102.

[14] Li, J., Bai, Y., Li, T. 2005. Highly Photoluminescent Cdte / Poly (NIsopropylacrylamide) Temperature-Sensitive Gels. Advanced Materials, 17,163 .

[15] Zhang, H., Edwards, E.W., Wang, D., Möhwald, H. 2006. Directing the Self-Assembly Of Nanocrystals Beyond Colloidal Crystallization. Phys. Chem. Chem. Phys., 8, 3288.

[16] Li, M.J., Zhang, J.H., Zhang, H., Liu, Y.F., Wang, C.L., Xu, X., Tang, Y., Yang, B. 2007. Preparation of Zno Doped Transparent And Fluorescent Composite Films By Sol-Gel Method. Adv. Funct. Mater, 17, 3650.

[17] Rosca, I.D., Watari, F., U.M. 2004. Microparticle Formation And Its Mechanism In Single And Double Emulsion Solvent Evaporation. J. Controlled Release, 99, 271.

[18] Fantini, D., Zanetti, M., Costa, L.G. 2006. Macromol. Rapid Comm., 27, 2038.

[19] Li, D., Xia, Y. 2004. Electrospinning of Nanofibers Reinventing The Wheel. Adv. Mater, 16, 1151.

[20] Long, J.X. 2013. The Preparation of Polyimide And Polyimide/Mental Nanofiber Via Electrospinning. Beijing University of Chemical Technology.

[21] Bai, J., Li, Y.X., Zhang, C.Q., Liang, X.F., Yang, Q.B. 2008. Novel Approach to Prepare Agcl/Pvp Nanocomposite Microspheres. Colloids and Surfaces A, 329,165.

[22] Wan, L.S., Xu, Z.K., Ke, B.B. 2006. Polyacrylonitrile-Based Electrospun Nanofibrous Materials. Macromol Rapid Commun, 27, 1533. 